\title{
Different sets of TaCKX genes affect yield- related traits in wheat plants grown in a controlled environment and in field conditions
}

\author{
Karolina Szala', Hanna Ogonowska', Boguslawa Lugowska², Barbara Zmijewska³ ${ }^{3}$ Renata Wyszynska ${ }^{4}$, \\ Marta Dmochowska-Boguta ${ }^{5}$, Waclaw Orczyk ${ }^{5}$ and Anna Nadolska-Orczyk ${ }^{1 *}$ (D)
}

\begin{abstract}
Background: TaCKX wheat gene family members (GFMs) encode the enzyme cytokinin oxidase/dehydrogenase $(C K X)$, which irreversibly degrades cytokinins. The genes are important regulators of cytokinin content and take part in growth and development, with a major impact on yield-related traits. The goal of this research was to test whether these genes might be differentially expressed in the field compared to laboratory conditions and consequently differently affect plant development and yield.

Results: We compared expression and crosstalk of the TaCKX GFMs and TaNAC2-5A gene in modern varieties grown in a growth chamber (GC) and in the field and looked for differences in their impact on yield-related traits. The TaNAC2-5A gene was included in the research since it was expected to play an important role in co-regulation of these genes. The range of relative expression levels of TaCKX GFMs and TaNAC2-5A gene among tested cultivars was from 5 for TaCKX8 to more than 100 for TaCKX9 in the GC and from 6 for TaCKX8 to 275 for TaCKX10 in the field. The range was similar for four of them in the GC, but was much higher for seven others and TaNAC2-5A in the field. The TaCKX GFMs and TaNAC2-5A form co-expression groups, which differ depending on growth conditions. Consequently, the genes also differently regulate yield-related traits in the GC and in the field. TaNAC2$5 A$ took part in negative regulation of tiller number and CKX activity in seedling roots only in controlled GC conditions. Grain number and grain yield were negatively regulated by TaCKX10 in the GC but positively by TaCKX8 and others in the field. Some of the genes, which were expressed in seedling roots, negatively influenced tiller number and positively regulated seedling root weight, CKX activity in the spikes, thousand grain weight (TGW) as well as formation of semi-empty spikes.
\end{abstract}

Conclusions: We have documented that: 1) natural variation in expression levels of tested genes in both environments is very high, indicating the possibility of selection of beneficial genotypes for breeding purposes, 2) to create a model of an ideotype for breeding, we need to take into consideration the natural environment.

Keywords: TaCKX, TaNAC, Wheat, Field, Laboratory conditions, Cytokinin, Phytohormone crosstalk, Grain yield

\footnotetext{
* Correspondence: a.orczyk@ihar.edu.pl

${ }^{1}$ Department of Functional Genomics, Plant Breeding and Acclimatization Institute - National Research Institute, Radzikow, 05-870 Blonie, Poland

Full list of author information is available at the end of the article
}

(c) The Author(s). 2020 Open Access This article is licensed under a Creative Commons Attribution 4.0 International License, which permits use, sharing, adaptation, distribution and reproduction in any medium or format, as long as you give appropriate credit to the original author(s) and the source, provide a link to the Creative Commons licence, and indicate if changes were made. The images or other third party material in this article are included in the article's Creative Commons. licence, unless indicated otherwise in a credit line to the material. If material is not included in the article's Creative Commons licence and your intended use is not permitted by statutory regulation or exceeds the permitted use, you will need to obtain permission directly from the copyright holder. To view a copy of this licence, visit http://creativecommons.org/licenses/by/4.0/ The Creative Commons Public Domain Dedication waiver (http://creativecommons.org/publicdomain/zero/1.0/) applies to the data made available in this article, unless otherwise stated in a credit line to the data. 


\section{Background}

Wheat is one of the most economically important cereal crops [1]. It can be cultivated in a wide range of environmental conditions and is rich in nutrition components. It provides approximately $20 \%$ of protein in the human diet [2]. The large, hexaploid genome, which is composed of three A, B and D diploid genomes, is a rich reservoir of genes determining yield-related traits [3]. However, the increase of wheat yield has remained moderate or even stagnate within the last two decades $[4,5]$. This cereal species is much less studied compared to rice and maize [6].

CKX GFMs encode the enzyme cytokinin oxidase/dehydrogenase (CKX), which irreversibly degrades cytokinins [7], and therefore strongly regulates cytokinin content in different organs of plants. Since cytokinins play a diverse role in plant development and affect a number of agriculturally important processes $[8,9]$, the $C K X$ genes influence yield-related traits. In cereals it was documented by means of silencing of selected $C K X$ in rice [10], in barley $[11,12]$ and in wheat [13] leading to an increased level of cytokinins, affecting yield components. The phytohormone also regulates changes in gene expression of cytokinin-induced genes to mediate its pleiotropic effect $[9,14]$.

The number of $C K X$ GFMs varies depending on species. In bread wheat 11 to 14 gene family members have been proposed [15-18]. TaCKX2 underwent gene duplication [15] and the resultant genes were assigned as TaCKX2.1 and TaCKX2.2 based on the Ensembl Plants database [19] and phylogenetic analysis by Ogonowska et al. [16]. The numbering of TaCKX GFMs was recently revised taking advantage of the most up-to-date databases (including IWGSC RefSeq v2.0), their homology with closely related monocot species including barley and diploid genome specificity [20]. Based on these data, there are $13 \mathrm{TaCKX}$ GFMs basically numbered as TaCKX1, TaCKX2.1, TaCKX2.2.1, TaCKX2.2.2, ТaCKX2.2.3, ТaCKХ3 (6), TaCKX4, TaCKX5, TaCKX7, TaCKX8 (11), TaCKX9 (10), TaCKX10 (9) and TaCKX11 (3), and 12 of them are allocated to each of the three chromosomes associated with the A, B and D genomes, giving the number of 35 homoeologous genes. The exception is TaCKX2.2.2 allocated only to $\mathrm{D}$ genome. This new numbering of the TaCKX genes is applied in this publication and the former numbers are shown in brackets.

The CKXs exhibit distinct patterns of organ- and development-specific expression. In our earlier research on $H \nu C K X$ in barley we found that expression patterns of the genes indicate their role in growth and reproductive development [21]. Therefore, the first step of our investigation of the $C K X$ genes in wheat was focused on expression specificity in different organs and different developmental stages [16]. Based on these results the genes were classified to four groups: i) leaf-specific
TaCKX9, TaCKX5, TaCKX4; ii) specific to inflorescence and developing spike TaCKX1 and TaCKX2; iii) seedling root-specific $\mathrm{TaCKX10}$, TaCKX7 and iv) expressed at various levels in all tested organs TaCKX11, TaCKX3, TaCKX8 [16]. The TaCKX GFMs co-operated inside and among organs. The effect of TaCKX1 silencing was further investigated in $7 \mathrm{DAP}$ (days after pollination) spikes [13]. Various levels of TaCKX1 silencing in $\mathrm{T}_{1}$ and $\mathrm{T}_{2}$ generations influenced different modes of co-expression with other TaCKX GFMs and parameters of yield-related traits. Only lines with strongly silenced TaCKX1 and associated with this strong down-regulated TaCKX11 and upregulated TaCKX2.1 and TaCKX9 were characterized as high yielding. The content of most of the cytokinins in spikes 7 DAP of silenced $\mathrm{T}_{2}$ lines significantly increased, and their interaction with other phytohormones was demonstrated. Each of the tested yield-related traits was regulated by various up- or down-regulated TaCKX GFMs and phytohormones.

Cytokinins regulate gene transcription in target organs and developmental stages through a wide range of transcription factors (TF) [22]. One of the largest groups of plant TFs involved in cytokinin-dependent regulation is the family of NAC (for NAM, ATAF, and CUC) TFs. It has been documented that NACs are involved in the regulation of important agronomic traits [23-26]. Overexpression of nitrate inducible wheat TaNAC2-5A increased root growth, the rate of nitrate uptake under hydroponic conditions and in the field increased grain yield, grain nitrogen $(\mathrm{N})$ concentration, and $\mathrm{N}$ harvest index under various $\mathrm{N}$ supply levels [23]. This gene was included in our research as an important regulator of cytokinin activity.

Experiments in controlled environments, in contrast to field conditions expose plants to constant irradiance during the day, constant temperature and artificial soil conditions. The growth parameters and the morphology of Arabidopsis plants, grown under artificial light, independent of the source, were significantly different compared with plants grown in natural conditions [27]. Distinct epistatic interactions on flowering time in barley were observed in field and in semi-controlled conditions [28]. Moreover, plant hormones differently regulated grain development in normal and under abiotic stress conditions [29-32]. It was also reported that yield stability genes are differentially expressed in the field compared to laboratory conditions [33].

Transgenic solutions to increase yield in wheat seemed to be very promising, but no transgenic cultivar has yet been approved [5]. On the other hand, natural variation is proposed as a profitable rich source of research objects to understand the responses mediated by jasmonate signalling [34]. We have been testing two alternative strategies of changing TaCKX-dependent cytokinin regulation in order to increase grain yield in wheat. One 
is based on the modifications of the TaCKX genes' expression via RNAi-based gene silencing [13]. The second one, presented here, explores the possibility of selection of natural variants differing in expression of TaCKX GFMs in wheat genotypes/breeding material. Differences revealed in co-regulation of yield-related traits by these GFMs in different environments, could lead to selection of appropriate genotypes in breeding programmes dedicated to specific environment. Therefore in the first part of the study we examined the range of expression of the TaCKX GFMs in 7 DAP spikes and in seedling roots among cultivars and breeding lines in a growth chamber and in the field. We analysed co-expression among themselves and with TaNAC2-5A and their influence on yieldrelated traits in both environments. In the next part of this research we will investigate how expression patterns of selected marker genes are inherited and whether they are consistent with inheritance of yield-related traits.

\section{Results}

\section{Expression of TaNAC2-5A in different organs of} developing wheat plants

The level of relative expression of TaNAC2-5A (NAC2) was highest in leaves, seedling roots and 0 DAP spikes, ranging from 0.015 up to 0.057 (Fig. 1). This wide range was mainly dependent on the large differences of expression of the gene among three tested cultivars. For two of them, the cultivars Ostka and Trappe, the expression levels of the $N A C 2$ in leaves was about three times higher than in Kontesa. Interestingly, opposite data of expression levels in the same cultivars were found in inflorescence, where they were several times lower.

There were no significant differences in expression of $N A C 2$ in 0 DAP spikes among cultivars. Expression levels of NAC2 in 7 DAP and 14 DAP spikes ranged from 0.005 to 0.01 . There were some differences between cultivars, but not between the first and the second spike or daily times of sample collection.

\section{Range of variability of expression of TaCKX GFMs and $N A C 2$ in growth chamber and the field}

Expression levels of TaCKX GFMs and NAC2 were measured in 7 DAP spikes of 34 breeding lines growing in a growth chamber (GC) and in the field. Highest, mean and lowest values of relative expression are presented in Fig. 2. The level of the CKX1 mean relative expression was higher in 7 DAP spikes from the GC (0.043) compared to the same material from the field (0.026). However the highest $(0.126)$ and the lowest $(0.016)$ values obtained for the same gene in the GC indicated a 7.88-fold difference (quotient of variability), while the values $(0.102$ and 0.006$)$ in the field showed 18.36-fold difference.

Similar to $C K X 1$, mean expression of $C K X 2.2 .1$ and NAC2 (TaNAC2-5A) were higher in the GC $(0.0038$ and $0.020)$ compared to the field $(0.0025$ and 0.015$)$. However for most of the TaCKX GFMs (CKX2.2.2, CKX3, CKX5, CKX8, CKX9, CKX10, CKX11) the mean values of expression were higher in the field compared to the GC. The range of expression variability between breeding lines in 7 DAP spikes growing in both conditions was similar for $C K X 2.2 .1, C K X 2.2 .2, C K X 5$ and $C K X 9$, and showed big differences for $C K X 1, C K X 3, C K X 4$, CKX5, CKX10, CKX11 and NAC2 in the field conditions. The highest variability between expression levels of the genes from the GC compared to the field ranging from 5 to 10 times was observed for $C K X 4, C K X 10$ and $C K X 11$. CKX1 was highly expressed followed by CKX11, CKX2.2.2 and CKX9 in both growing conditions. The mean level of NAC2 expression was similar to CKX11 in the field (0.016 and 0.015 respectively) and two times

TaNAC2-5A

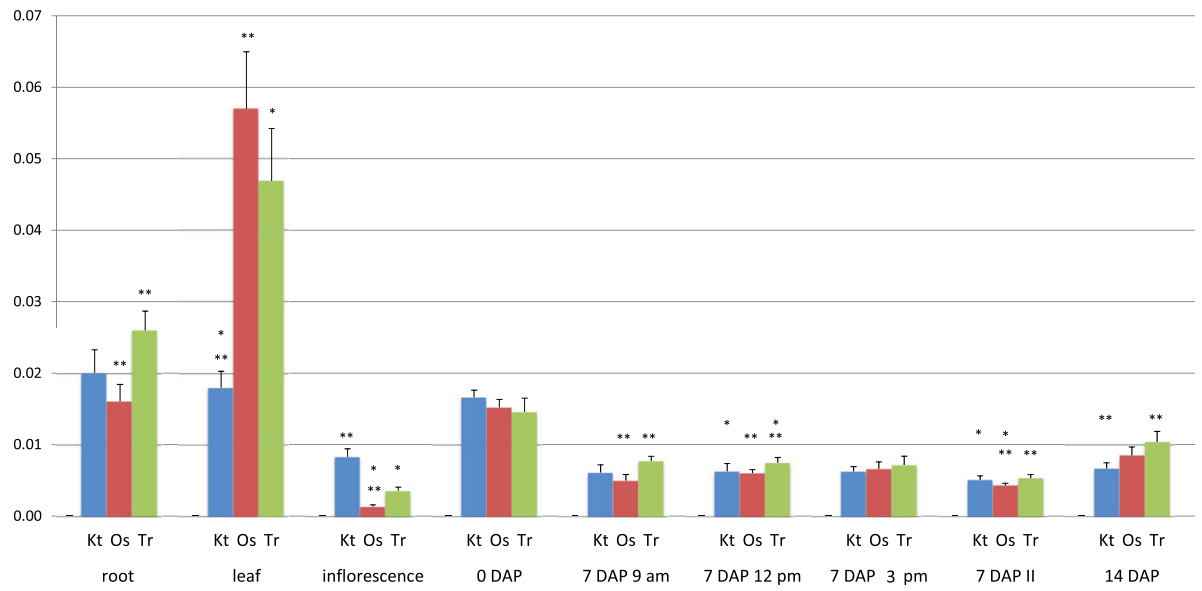

Fig. 1 Specificity of expression of TaNAC2-5A gene in developing organs of wheat. ** - significant at $p \leq 0.01$; * significant at $p \leq 0.05$; II second spike 

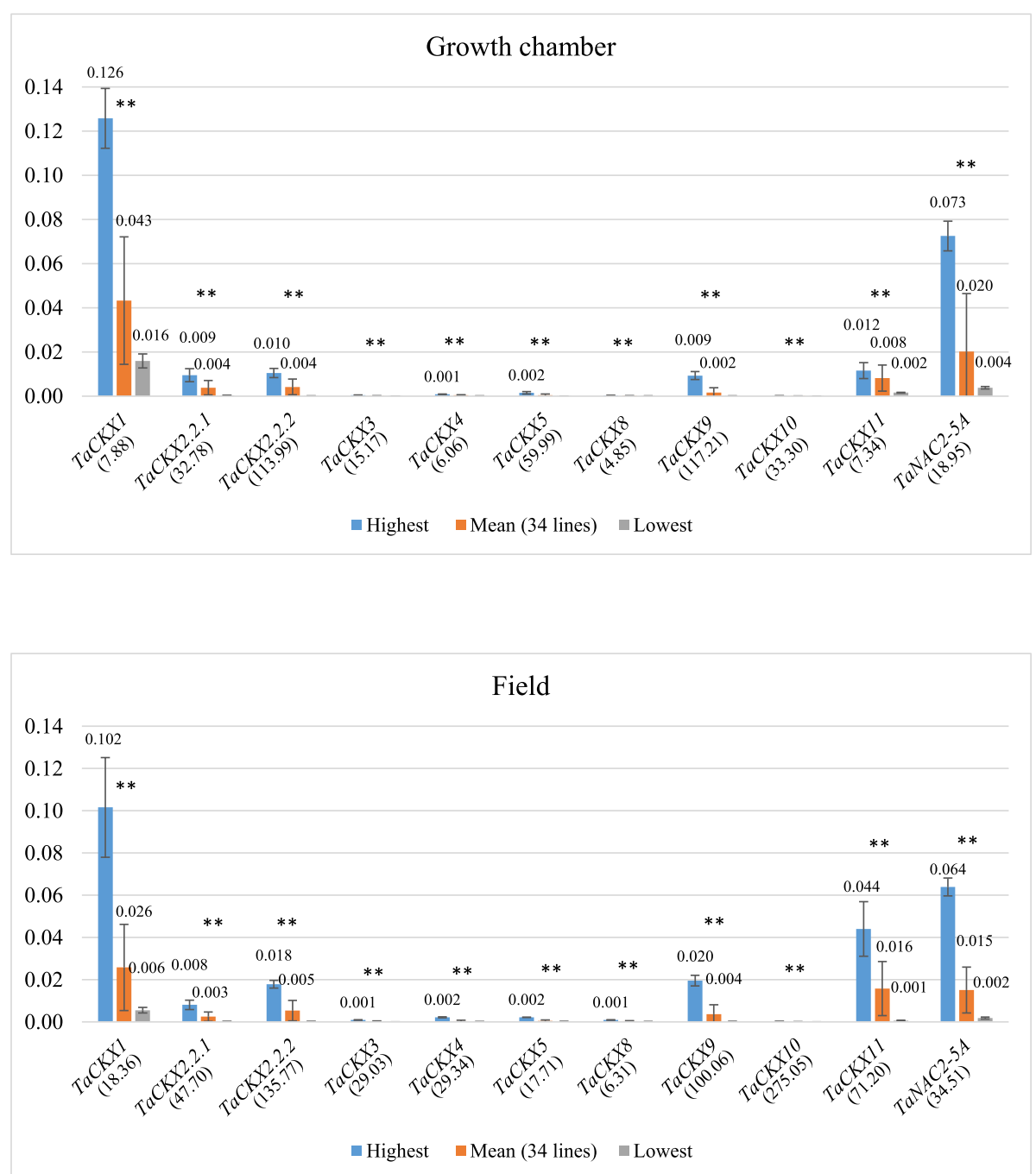

Fig. 2 Highest, mean and lowest data of expression levels of TaCKX GFMs and NAC2 in 7 DAP spikes of 34 breeding lines growing in growth chamber and in the field. ${ }^{*}$ - significant at $p \leq 0.01$ (between highest and lowest). (...) - quotient of variability

higher in the GC (0.020 and 0.008 respectively). Standard deviation of gene expression levels among three biological replicates were higher in spikes from the field compared to the GC.

\section{Co-expression of TaCKX GFMs in growth chamber and the field}

Correlation between expression levels of various TaCKX GFMs was different in both growth conditions, GC and the field (Fig. 3, Table S2). Since breeding lines were grown on two different fields of two Plant Breeding Companies Strzelce (S) and Danko (D), correlations are presented for both conditions together $(S+D)$ and separately ( $\mathrm{S}$ or $\mathrm{D})$. Generally, in field conditions the groups of mutually correlated genes were larger than in GC conditions and co-expression of a few of them was specific to the field ( $\mathrm{S}$ or $\mathrm{D}$ ). According to expression specificity, the $\operatorname{TaCKX}$ GFMs were divided into four groups. The first one contained the genes specifically expressed in developing spikes; the genes from the second group were expressed in seedling roots; the third were specific in younger plant organs from seedling roots to 0 DAP spikes and the fourth to all organs tested.

Expression level of spike specific CKX1 significantly correlated with expression levels of $C K X 2.2 .2$ and CKX11 in the GC, but no correlation with others was found in spikes from both fields. Otherwise $C K X 5$ showed specific negative co-expression with $C K X 1$, when spikes were grown on D field. $C K X 2.2 .2$, whose expression correlated with $C K X 1$ in the $\mathrm{GC}$, was strongly correlated with $C K X 3, C K X 10$ and $C K X 8$ in both fields, and individually with $C K X 2.2 .1$ in S field. $C K X 2.2 .1$ was co-expressed with $C K X 5$ in both growing conditions. The gene was also co-expressed with $C K X 3, C K X 8$ and 


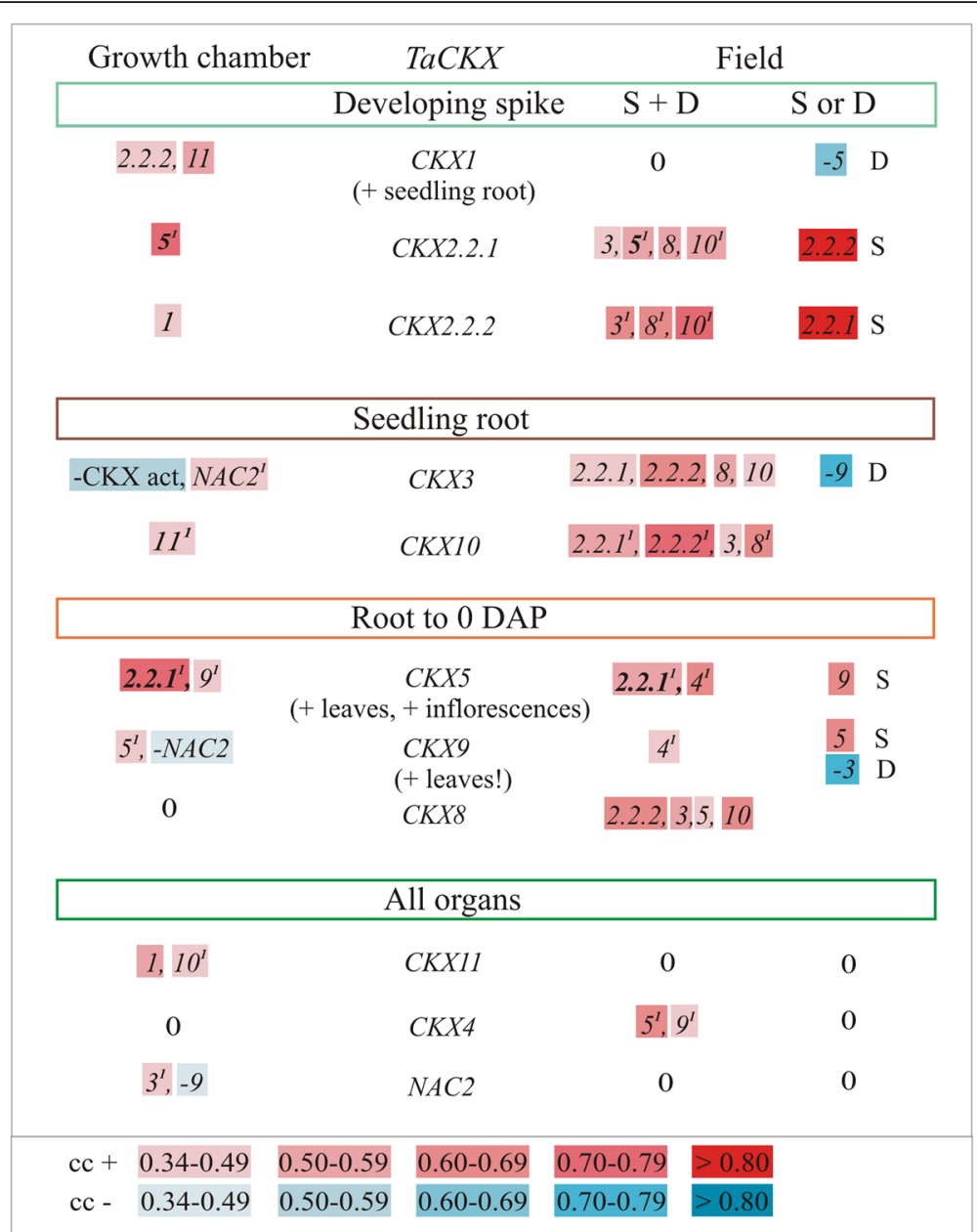

Fig. 3 Co-expression of TaCKX GFMs and NAC2 in 7 DAP spike from the growth chamber and from the fields of Strzelce (S) and Danko (D) together $(S+D)$ or separately ( $\mathrm{S}$ or $\mathrm{D}$ ) based on correlation coefficient (cc). All $c c$ are significant at $p \leq 0.05$. Gradation of positive $c c$ in red and negative cc in blue; " 0 " - lack of correlation; ${ }^{1}$ - non-parametric; bold - cc in the growth chamber and in the field

CKX10 in both fields and individually with CKX2.2.2 in $\mathrm{S}$ field. Expression of root-specific $C K X 3$ from the second group of genes positively correlated with expression of $N A C 2$ and negatively with CKX activity in the GC and with CKX2.2.1, CKX2.2.2, CKX8 and CKX10 in the fields as well as strongly negatively with $C K X 9$ in $\mathrm{D}$ field. Another rootspecific, CKX10, exclusively correlated with CKX11 in the GC and with $C K X 2.2 .1, C K X 2.2 .2$, and $C K X 8$ in the fields as in the case of $C K X 3$. Moreover, expression level of both, $C K X 3$ and $C K X 10$ showed a mutual correlation in the fields conditions. The CKX5, which was strongly expressed in younger organs, especially in seedling roots, leaves and inflorescences, co-expressed with $C K X 2.2 .1$ in both conditions, with $C K X 9$ in GC and with CKX4 in the fields as well as with $C K X 9$ in $\mathrm{S}$ field. Expression of the next one from this group, $C K X 9$, which was especially high in leaves, was positively correlated with $C K X 5$ and negatively with $N A C 2$ in GC but positively with $C K X 4$ in both fields, and positively with $C K X 5$ in $\mathrm{S}$ field but negatively with $C K X 3$ in $\mathrm{D}$ field. No correlation was found between expression of CKX8 and any of the tested genes in GC. However, the CKX8 was positively correlated with $C K X 2.2 .2$, CKX3, CKX5 and CKX10 in both fields conditions.

The last group represented genes expressed in all organs. Interestingly, expression of CKX11 correlated with expression of $C K X 1$ and CKX10 in GC. There was no correlation of this gene with others in the field conditions. Conversely, the level of CKX4 expression positively correlated with CKX5 and CKX9 in both fields, but there was no correlation in GC. There was a positive correlation between expression of $N A C 2$ and $C K X 3$ and a negative correlation with $C K X 9$ in GC. No correlation of $N A C 2$ with others was found in the field.

Expression of TaCKX GFMs and NAC2 and CKX activity in 7 DAP spikes differently affect yield-related traits in both growth conditions

In the GC, plant height and TGW were positively correlated with CKX activity in 7 DAP spikes (Fig. 4, Table 


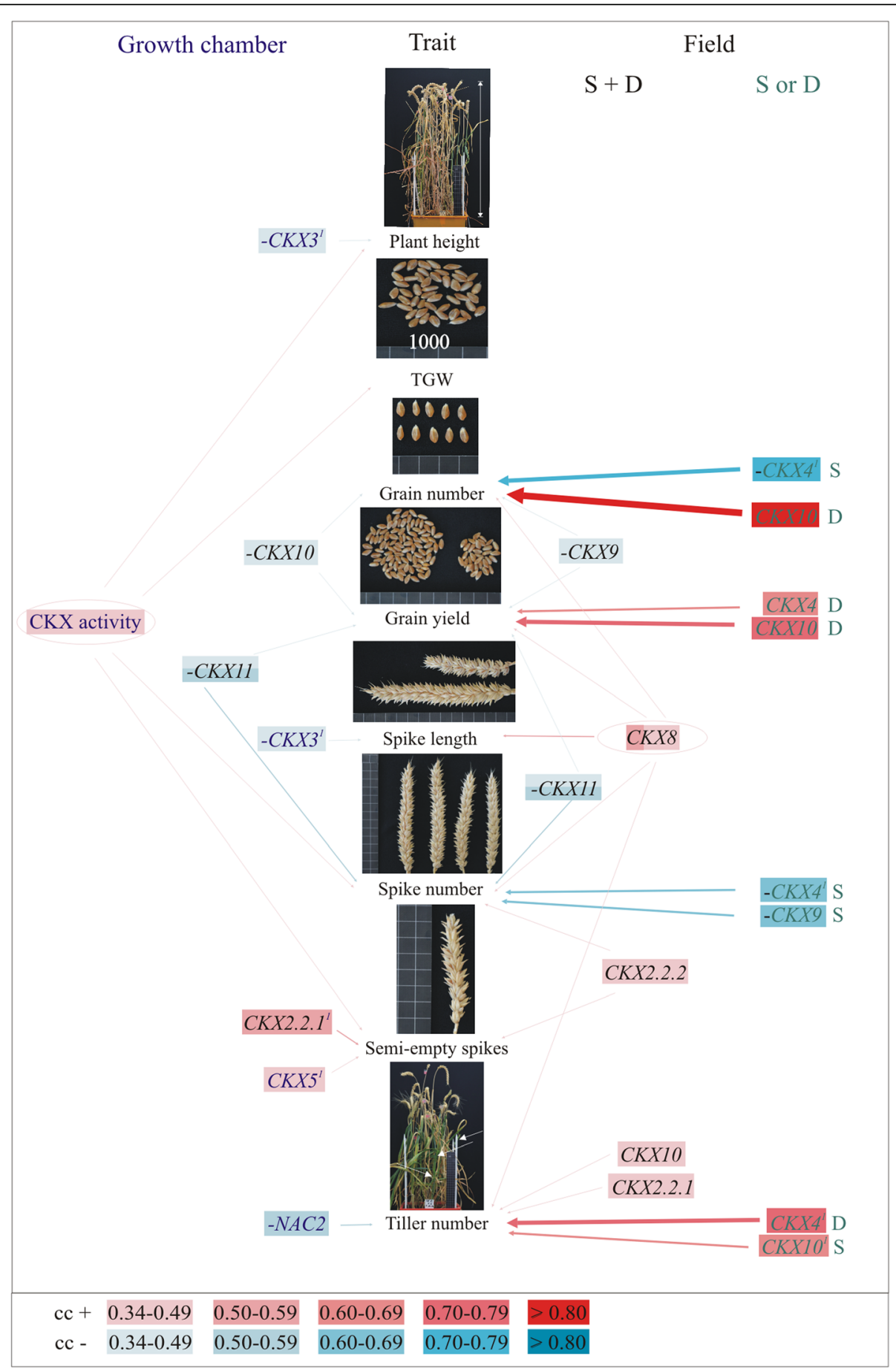

Fig. 4 Interconnections between TaCKX GFMs and NAC2 expression, and CKX activity in 7 DAP spikes from the growth chamber (left) and the fields (right) with yield-related traits based on significant cc. The cc from the fields of Strzelce $(S)$ and Danko (D) counted together $(S+D)$ or separately (S or D). Gradation of positive cc in red and negative cc in blue; ${ }^{1}$ - non-parametric analysis; "-"negative correlation

S3). However, the plant height and the spike length were negatively correlated with $C K X 3$. Enzyme activity was also positively correlated with number of spikes and number of semi-empty spikes. Moreover, the number of semi-empty spikes is positively correlated with expression of $C K X 2.2 .1$ and $C K X 5$, and tiller number is negatively correlated with $N A C 2$. Grain number and grain yield in the same growth conditions are negatively correlated with $C K X 10$. Otherwise, in the fields conditions both grain number and grain yield are negatively 
correlated with expression of $C K X 9$, and positively correlated with $C K X 8$, but the grain yield is negatively correlated with CKX11 (spikes from both fields). Additionally grain number is strongly negatively correlated with $C K X 4$ expression in spikes from $\mathrm{S}$ field and very strongly positively correlated with $C K X 10$ in case of spikes from D field. Otherwise grain yield is positively regulated by $C K X 4$ and $C K X 10$ in case of $\mathrm{D}$ field. The CKX8 expressed in spikes from both fields is not only positively correlated with grain number and grain yield but also with spike length, spike number and tiller number. Both spike number and number of semi-empty spikes are positively correlated with $C K X 2.2 .2$ measured in spikes collected from both fields. Individually, spike number is negatively correlated with CKX4 and CKX9 only in case of spikes from $S$ field and tiller number is positively correlated with CKX4 and CKX10 measured in spikes from $\mathrm{D}$ and $\mathrm{S}$ fields respectively.

\section{Expression of TaCKX GFMs and NAC2 and activity of CKX in seedling roots affect yield-related traits}

Expression of CKX1 in seedling roots was positively and strongly correlated with $C K X 3, C K X 5, C K X 8$ and $N A C 2$ (Fig. 5a, Table S4). There was no correlation between CKX10 and CKX11 and other genes expressed in this organ.

CKX3 expressed in seedling roots is the most frequently interacting gene with yield-related traits in the GC (Fig. 5b, Table S4). Its expression positively

\section{A. I $\quad$ CKXI $\rightarrow C K X 3, C K X 5, C K X 8, N A C 2$ \\ II $C K X 10, C K X 11$ - 0}

B.

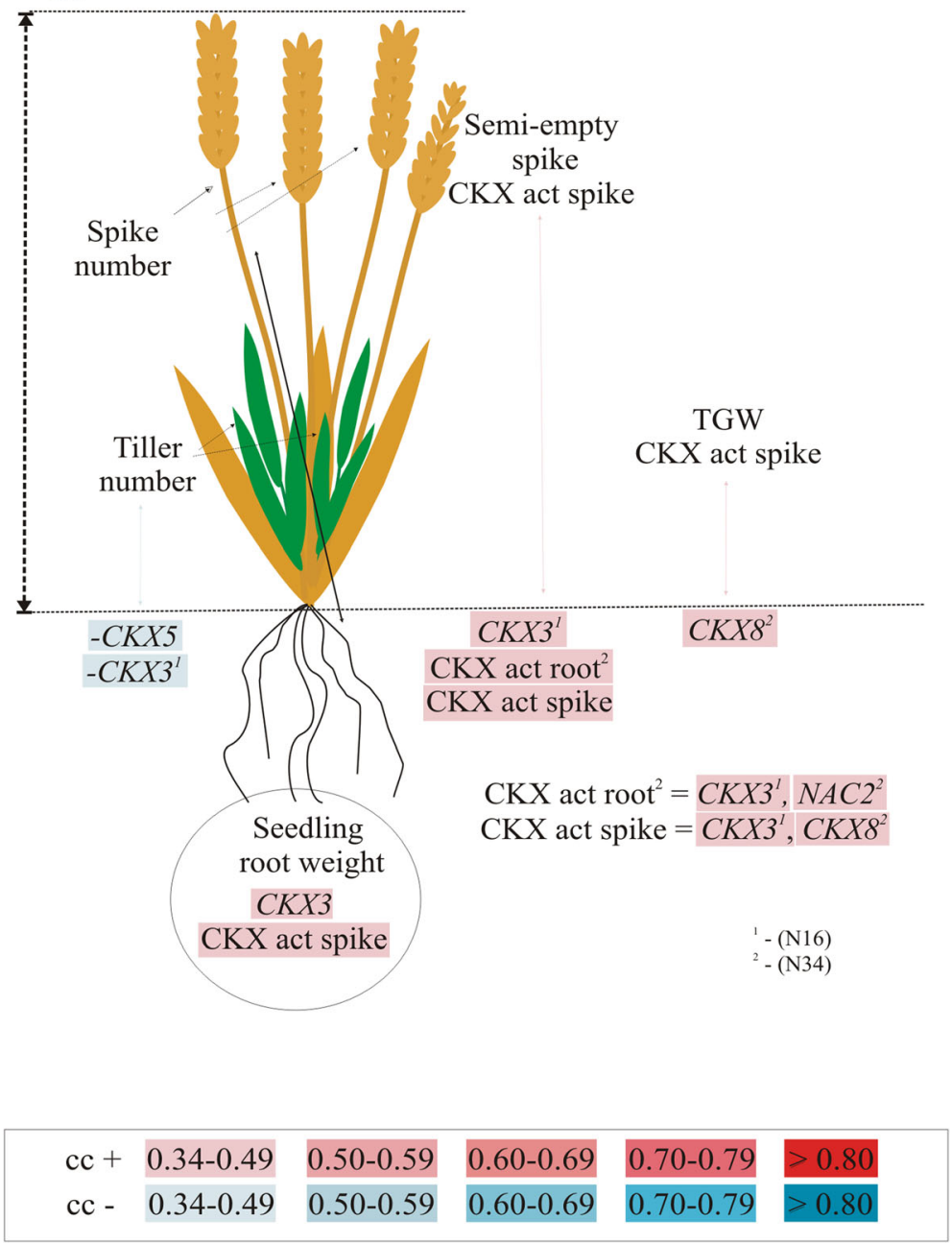

Fig. 5 Influence of TaCKX GFMs and NAC2 expression, and CKX activity (CKX act) in seedling roots on seedling root weight and yield-related traits. Coexpression of TaCKX GFM and NAC2 in seedling roots (a) and their cooperation with above ground yield-related traits (b) based on significant $c c(p \leq 0.05)$. 0 - no correlation; N16, N34 - cc for 16 and 34 breeding lines respectively; gradation of positive cc in red and negative cc in blue 
correlated with seedling root weight and semi-empty spikes, but negatively with tiller number. Positive correlation of the CKX3 is accompanied by CKX activity in spikes, and in the case of semi-empty spikes, additionally with CKX activity in roots. Moreover, CKX3 and CKX8 are positive regulators of spike CKX activity and, with $N A C 2$, positively correlated with CKX activity measured in seedling roots. Expression of $C K X 8$ is positively correlated with CKX activity in spikes and TGW. Expression of $C K X 5$ is negatively correlated with tiller number.

\section{Discussion}

\section{TaNAC2-5A is expressed in different organs of wheat} plants

TaNAC2-5A (NAC2) was included into this research on regulation of yield-related traits of wheat by the TaCKX gene family as a possible important regulator of these genes and the corresponding processes. This nitrateinducible $N A C 2$ encoding gene was reported by $\mathrm{He}$ et al. [23] to be an important regulator of root growth and wheat yield. To relate $N A C 2$ expression to our previous research on expression specificity of $\operatorname{TaCKX}$, we performed expression experiments similar to those we did with TaCKX [16]. Relative expression levels of NAC2 in roots, leaves and 14 DAP spikes reported by $\mathrm{He}$ et al. [23] were comparable with our results. The NAC2 is also expressed in organs newly tested by us: inflorescence, 0 DAP and 7 DAP spikes, but at a level lower than for 14 DAP spikes. Generally, the gene is expressed in all organs tested. We also demonstrated that, especially in the case of leaves, inflorescence and root expression, levels of NAC2 differed considerably between cultivars.

\section{Large differences in TaCKX GFMs and NAC2 expression levels among breeding lines allow them to be selected for breeding purposes}

The differences between the highest and the lowest expression levels of tested TaCKX GFMs and NAC2 in breeding lines were significant and ranged from several to over a hundred times. These differences were much bigger for six of the genes expressed in 7 DAP spikes growing in the field compared to GC. This is consistent with the observation that stress inducible genes were expressed more strongly in field than in laboratory conditions [33]. These differences in expression might be the result of allelic variation among the tested genes, their genetic background and cross-talk with environments. Allelic variation among $T a C K X$ family genes corresponding with yield-related traits was reported by others [35-37]. The data indicate the possibility of using this parameter for selection in the breeding process.
Selected TaCKX GFMs are differentially expressed in field and GC conditions

The similar range of expression variability among breeding lines for $C K X 2.2 .1, C K X 2.2 .2, C K X 8$ and $C K X 9$ in the $\mathrm{GC}$ and in the field might indicate that expression levels of these genes are independent of growth conditions. The data indicating stable expression being maintained by these genes in changing conditions are in agreement with newly published results of TaCKX1 silencing in the case of both $C K X 2.2$ [13]. There was strongly decreased expression of $T a C K X 1$ gene in silent lines while expression of $C K X 2.2$ remained at the same level. Otherwise, the impact of unstable field growth conditions on expression of $C K X 1, C K X 3, C K X 4, C K X 5$, CKX10, CKX11 and NAC2 was significant. Indeed, TaCKX1 silencing caused strong down-regulation of expression of $C K X 11$ and up-regulation of CKX5 [13]. It may be dependent on the same feedback mechanism between induction of expression of selected $C K X$ genes by cytokinin and their function [38]. It was already documented that constant irradiance during the day in controlled environments and lack of light-day transitions may significantly influence plant phenotype [27]. Consequently, many group of genes determining yield in normal and stress conditions, genes encoding chloroplast located proteins or shade avoidance (including genes involved in hormonal regulation, light and flowering) are differentially expressed in growth chamber versus field conditions [33, 39]. Expression of $C K X$ as well as biosynthetic IPT genes might also be regulated by the levels of available macronutrients, such as nitrate and phosphate [40], and biotic/abiotic stress conditions in the field.

\section{TaCKX GFMs and NAC2 in field or controlled environments form different co-expression groups}

The TaCKX GFMs and NAC2 are predominantly coexpressed in the GC and in the field in different groups. Only two of them co-work with each other in both conditions: CKX2.2.1 expressed in developing spikes and CKX5 highly expressed in seedling roots, inflorescences and leaves [16]. This cooperation may indicate their importance in regulation of cytokinin level between roots and spikes during plant growth and development independently of growth conditions.

What is remarkable, CKX1 highly expressed in developing spikes and roots, and $C K X 11$ and $N A C 2$ expressed in all organs formed co-expression groups with other genes only in GC conditions. It does not mean that the genes are not susceptible to different nutrition conditions since $N A C 2$ was shown to be inducible by nitrate [23]. However, it is possible that expression of these genes might be independent of main environmental factors as the source of light and light-day transitions as well as changing temperatures and soil moisture. CKX11 
highly expressed in all organs, co-worked with $C K X 1$ and CKX10 in the GC conditions but not in the field, although their expression in the field was seven times higher. This strong co-regulation of CKX11 with CKX1 was already observed in our research with silencing the TaCKX1 performed in GC conditions [13]. Conversely CKX4 expressed in all organs and CKX8 expressed in younger organs, which did not show any co-expression with others in GC, were co-regulated by others in the field. Therefore, these genes might take part in regulation of cytokinin content by involving others in more stressful conditions.

Significantly larger were groups of $T a C K X$ genes coexpressed in the unstable, field conditions. CKX8 expressed in younger organs was the most frequently co-working with others, mainly those expressed in developing spikes and roots. However, the gene co-works with CKX5 belonging to the same expression group, which might link coregulation of expression of other genes in both growth conditions. Expression of CKX2.2.1 and 2.2.2 specifically expressed in developing spikes is correlated with the same root-specific CKX3 and $C K X 10$ as well as $C K X 8$ specific to younger organs but only in field conditions. Both $C K X 2.2$ genes correlate with different genes in GC, CKX2.2.1 with $C K X 5$ and CKX2.2.2 with $C K X 1$, which might indicate their different detailed functions (discussed below).

Both root-specific $C K X 3$ and $C K X 10$ are co-regulated with both, spike-specific $C K X 2.2$ and $C K X 8$ specific to all organs, and each other in the field, but in GC these genes specifically co-work with GC-specific $N A C 2$ and CKX11 respectively.

Yield-related traits are regulated by different TaCKX GFMs and $N A C 2$, depending on the natural or controlled environments

As in the case of co-expression, more $C K X$ genes were involved in regulation of yield-related traits, especially grain number, grain yield, spike number and tiller number in the field conditions than in GC. The most active in the field was CKX8 expressed in all organs coregulating with $C K X 9$ grain number, with $C K X 9$ and CKX11 grain yield, with CKX11 spike number, with CKX2.2.1 and CKX10 tiller number and as a single spike length. In most cases these traits are positively regulated by $C K X 8$ but negatively by co-expressed $T a C K X$ genes, which indicates their predominant role in maintaining hormonal homeostasis. Interestingly some traits like grain number, grain yield, spike number and tiller number are strongly regulated by $C K X 4$ and $C K X 10$ but this regulation is field dependent. Presumably both genes are strongly dependent on environmental conditions since both research fields differ in type of soil and weather conditions.
The same traits, grain number and grain yield, are regulated in GC negatively by CKX10. None of the genes regulates the same yield-related trait in both growth conditions. Similarly as in the study by Jablonski et al. [13], grain yield and spike number in GC were negatively regulated in 7 DAP spikes by $C K X 11$ which, in cooperation with $C K X 2.1$, influenced down-regulation of free base, active trans-zeatin (tZ) and $N^{6}$-( $\Delta 2$-isopentenyl) adenine (iP) but up-regulation of benzyladenine (BA). In the same research, spike number in GC-grown, control plants was regulated by $C K X 2.2 .1$ as well, which together with $C K X 1$ and $C K X 5$ up-regulated $\mathrm{tZ}$ and $\mathrm{iP}$ and downregulated BA. Some of our data do not appear comparable, because in Jablonski et al. [13] only one cultivar with a restricted number of TaCKX genes and yieldrelated traits was tested. However, it was shown that the genes form different co-expression groups in 7 DAP spikes of TaCKX1 silent and not-silent plants whereby they control the content of various cytokinins and determine final yield-related traits. Different coregulation of both CKX2.2 genes in the GC and the field resulted in their various impact on yield-related traits. $C K X 2.2 .1$ together with CKX5 is involved in formation of semiempty spikes in GC but $C K X 2.2 .2$ alone has such involvement in the field. $C K X 2.2 .1$ together with $C K X 8$ and $C K X 10$ positively regulates tiller number in the field as well. None exhibits the same role in the GC and in the field. Zhang et al. [35] reported that TaCKX6, renamed by [20] as TaCKX2.2.1-3D, is an orthologue of OsCKX2 regulating rice yield [10]. The cultivar, which has an 18-bp deletion, showed decreased expression of this gene, which was associated with a greater TGW than in other haplotypes. There is a small incompatibility in maximum transcript level, which was reached at 8 DAP in the study by Zhang et al. [35] and was increasing from 0 DAP through 7 DAP and reaching the maximum at 14 DAP in our research (later stages were not tested). This increasing expression in developing spikes up to 14 DAP better explains the possible role of the gene in grain filling, which is an essential factor of greater TGW. Besides this, we did not find any correlation of expression of this gene with TGW, although its expression together with CKX5 positively influenced formation of semi-empty spikes in GC and with two others tiller number in the field. Therefore the joint effect of TaCKX family genes on regulation of yield-related traits depending on growth conditions is important. However, expression of TaCKX2.2.1-3A (originally TaCKX2.4) reduced by RNAi [41] showed a strong correlation with grain number in $\mathrm{T}_{3}$ due to an increased grain number per spike. Similar result is obtained in our research, in which CKX2.2.1 together with CKX5 positively regulates formation of semi-empty spikes in GC, although the same trait was regulated by $C K X 2.2 .2$ in the field. Moreover, 
function of $T a C K X 2.2 .1-3 A$ is closer to their orthologue OsCKX2 in rice, where is also associated with grain number [10]. According to $\mathrm{Li}$ et al. [41], different functions of TaCKX2.2.1-3D and TaCKX2.2.1-3A genes are the result of different locations on chromosomes $3 \mathrm{D}$ and $3 \mathrm{~A}$ respectively. We suppose that these differences might be additionally modulated by possible individual co-operation with other genes within an individual genetic background.

There were two more characterized TaCKX family genes, both demonstrated as determinant of yieldrelated traits. Isolated by $\mathrm{Lu}$ et al. [36], the novel allele TaCKX6a allocated to the TaCKX2.1 gene family [16] and renamed as TaCKX2.1-3D [20] showed, as in our earlier research [13], significant correlations with grain size, weight and grain filling rate in recombinant inbred lines. In the second report one out of two alleles of TaCKX4 found by Chang et al. [37] was positively associated with chlorophyll content and grain yield. The authors did not show any expression data. According to our research, the gene is expressed in all tested organs of developing wheat plant in the GC, showing higher expression in leaves, but their expression was low compared to others [16]. We did not find any correlation of their expression with yield-related traits in the CG. However we found higher expression of this gene in field conditions comparing to the GC as well as their strong correlations with yield-related traits, which was dependent on the individual field conditions.

\section{Selected TaCKX GFMs and NAC2 expressed in seedling roots regulate some yield-related traits}

As already reported [16], CKX1 expressed in seedling roots forms a co-expression group with $C K X 3, C K X 5$, CKX8 and NAC2 in the same organ. Moreover, the TaCKX GFMs and NAC2 gene expressed in seedling roots formed different co-expression groups with that expressed in developing spikes. It means that the genes also participate in coordinated development of the above ground part of the plant, which is dependent on growth conditions. Such cooperation of TaCKX GFMs is obvious, since they regulate CKX enzyme activity, which inactivates cytokinins, plant hormones known to act opposingly in shoot and root growth [42]. An example is $C K X 3$, highly expressed in seedling roots, which is positively correlated with seedling root weight but negatively correlated with plant height and spike length. $N A C 2$, controlling with $C K X 3$, CKX activity in roots, when expressed in spikes, negatively correlated with tiller number. Moreover, $C K X 3$ with $C K X 5$ expressed in roots negatively regulated tiller number, but $C K X 3$ with CKX activity in roots positively determined formation of semi-empty spikes, and CKX8 expressed in roots positively influenced TGW. Such cooperation of seedling root expressing genes with those expressed in organs from the aerial part of wheat plants and their influence on yield traits was also documented in our earlier research $[13,16]$.

Generally, overexpression of $C K X$ in roots of other plant species leads to enhanced root growth by reduction of cytokinin levels [43-46]. By contrast, an elevated cytokinin level inhibits root growth but promotes shoot growth in rice [47]. Among the CKX GFMs in rice, Gao et al. [48] indicated a key role of OsCKX4 in the initiation of crown roots by interaction between auxin and cytokinin biosynthesis. Mao et al. [49] underlined the role of NAC transcription factors on rice root development. Overexpressed OsNAC2 increased expression of several IPT, decreased OsCKX4 and OsCKX5 and increased cytokinin level. Therefore OsNAC2 stimulated cytokinin accumulation by promotion of cytokinin biosynthesis and repression of $C K X$ expression. This was affected by the binding of OsNAC2 to the promoter of OsCKX4. In our research, wheat TaCKX4 orthologous to OsCKX4 was not found to correlate with seedling root weight, possibly because of its very low level of expression in roots as well as other organs of wheat plants. However, we showed strong correlations between the CKX4 gene expression and yield-related traits, which was dependent on field conditions. The second orthologue of rice, TaCKX5, together with $C K X 3$, both expressed in roots, was negatively correlated with tiller number, but the same trait was negatively influenced by $N A C 2$ expressed in spike.

\section{Conclusions}

It was previously indicated that yield stability genes are differentially expressed in the field compared to growth chambers [33]. These differences might mean that many genetically modified plants carrying single, agronomically important genes cannot be applied in practice $[5,50]$. Therefore, to indicate which $T a C K X$ family genes might be applicable in breeding programmes, it is important to characterize them in both environments, laboratory conditions and in the field. Indeed, we found large differences in the levels of expression, crosstalk of those genes and correlations with agronomic traits in modern varieties and breeding lines dependent on the environmental conditions. We have documented that to create a model of an ideotype for breeding, we need to take into consideration the field environment. Natural variation in expression levels of tested genes, especially in the field, was very high, indicating the possibility of selection of beneficial genotypes/phenotypes for breeding purposes. Such selection based on variable expression of selected genes, resulting from mutations in the tested genetic background and cross-talk with others, can be carried out among recent breeding material in their field environments. We suppose that combining beneficial genotypes selected in such environments could help breeders to exploit available genetic variation and optimize further yield potential in their 
regional soil and climate conditions. Therefore, the next step of our research is to show if and how expression of TaCKX GFMs is inherited in subsequent generations.

\section{Methods}

\section{Plant material}

The experimental plant material was collected from 34 breeding lines and cultivars of common wheat (Triticum aestivum L.) delivered by two Plant Breeding Companies: Strzelce Ltd., Co. - IHAR-PIB Group (23 breeding lines) and Danko Hodowla Roslin Ltd. (11 breeding lines). Selected genotypes differed in productivity and other interesting breeding features, such as resistance to diseases, plant height, protein content (including gluten), and starch in grain or seed hardness. The plants were grown in two different growth conditions: in a growth chamber and in a field.

Field conditions: The field experiments were conducted on two experimental fields of Strzelce Company (Konczewice) and Danko Company (Choryn). The first is located in the north-central part of Poland (KujawskoPomorski region) and the second in the west-central (Wielkopolska region), about $150 \mathrm{~km}$ from each other. Experimental plots were $5 \mathrm{~m}^{2}(=5 \times 1 \mathrm{~m})$ and 400 grains were sowing per $1 \mathrm{~m}^{2}$. White beet and rape were the forecrops respectively. Nitrogen was applied twice at the beginning of spring vegetation and at the beginning of stem elongation in two doses $\left(100-120 \mathrm{~kg} \mathrm{ha}^{-1}\right.$ total). The soil was also fertilized by $20-50 \mathrm{~kg} \mathrm{ha}^{-1}$ phosphorus and $50-80 \mathrm{~kg} \mathrm{ha}^{-1}$ potassium, depending on the field requirements. Humidity and thermal conditions during the experiment are shown in Fig. 6. Water deficiency was supplemented when needed.

Germination of seeds and growth chamber conditions were performed as earlier reported [16]. The 5-days seedlings were replanted into pots with soil after earlier collection of seedling roots, which were cut $3 \mathrm{~mm}$ from embryo axis. The tissue samples: 5-day-old seedling roots and the middle parts of first spikes 7 days after pollination (7 DAP) were collected in three biological replicates from the growth chamber and from the field. In both environments spikes with the first 1-3 sticking out anthers from spikelets in the middle part were marked and collected after 7 days. Three biological replicates were selected randomly out of six in the growth chamber (one spike each) and out of whole plots in the field (three spikes each). These in the field were marked the same day and collected 7 days later. All these samples were started to collected at 9:00 am. Additional tissue samples used for testing $N A C 2$ expression were collected from three cultivars of common wheat: Kontesa, Ostka and Trappe according to Ogonowska et al. [16].

\section{RNA extraction and CDNA synthesis}

Total RNA from 5-day-old seedlings' roots and 7 DAP spikes was extracted using TRI Reagent (Sigma-Aldrich) according to the manufacturer's protocol. Purification of RNA and cDNA synthesis was performed as described in Ogonowska et al. [16].

\section{Quantitative RT-qPCR}

RT-qPCR analysis was performed for 11 target genes: TaCKX1 (JN128583), TaCKX2.2.1 (FJ648070)/TaCKX2.2.2 (GU084177), ТaCKX3 (former TaCKX6) (JN128587), TaCKX4 (JN128586), TaCKX5 (Lei et al. 2008), TaCKX8 (former TaCKX11) (JN128592), TaCKX9 (former TaCKX10) (JN128591), TaCKX10 (former TaCKX9) (JN128590), TaCKX11 (former TaCKX3) (JN128585), TaNAC2-5A (AY625683). Sequences of primers designed for the genes tested are shown in Table S1. RT-qPCR reactions were assayed as described in Ogonowska et al. [16]. Each reaction was performed in three biological and three technical replicates. The two standard curves method with

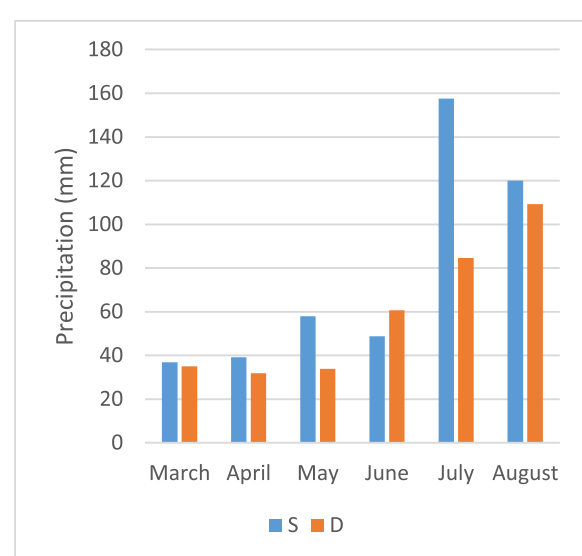

(A)

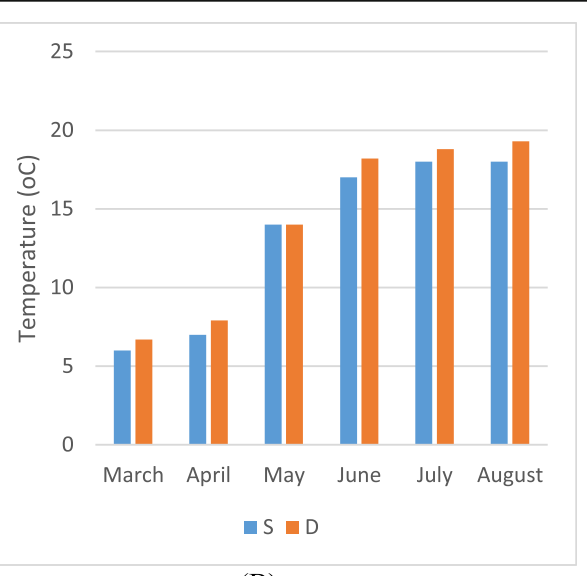

(B)

Fig. 6 Weather conditions for particular months in two Plant Breeding Companies, Strzelce (S) and Danko (D): total precipitation (a); mean air temperature (b) 
ADP-ribosylation factor (Ref 2) as a normalizer [51] was used for calculation of genes expression. Relative expression for each of the $\operatorname{TaCKX}$ family gene was calculated in relation to the control cultivar Ostka set as 1.00 .

\section{Analysis of CKX activity}

Measurements of CKX enzyme activity were performed in the same samples subjected to $\operatorname{TaCKX}$ gene expression analysis according to the procedure developed by Frebort et al. [52] and optimized for wheat tissues. The procedure is described in Zalewski et al. [12]. Concentration of the product was determined at the absorption spectrum ranged from $230 \mathrm{~nm}$ to $550 \mathrm{~nm}$. The total protein concentration was estimated based on the bovine serum albumin standard curve according to the Bradford procedure [53].

\section{Measurement of yield-related traits}

Morphometric measurement of yield-related traits of selected genotypes was performed. The described traits were: plant height, tiller number, spike number, semiempty spike number, spike length, grain number, grain yield, TGW and seedling root weight.

\section{Statistical analysis}

Statistical analysis was done using Statistica 13 (StatSoft) software. The normality of data distribution was tested using the Shapiro-Wilk test. The significance of changes was analysed using analysis of variance (ANOVA) and post-hoc tests. Correlation coefficients were determined using parametric correlation matrices (Pearson's test) or a nonparametric correlation (Spearman's test).

\section{Supplementary Information}

The online version contains supplementary material available at https://doi. org/10.1186/s12870-020-02713-9.

Additional file $\mathbf{1}$ Table $\mathbf{S 1}$. Sequences of primers designed for amplification of the genes. Table S2. Correlations among expression of TaCKX GFMs and NAC2 in 7 DAP spikes growing in growth chamber (A) and in the field (B) and in seedling roots (C). '- non-parametric analysis; bold* ${ }^{*}$ significant correlation at $p \leq 0.05$. Table S3. Correlations among expression of TaCKX GFMs and NAC2 in 7 DAP spikes from the growth chamber (A) and the field (B) on yield-related traits. ${ }^{1}$ - non-parametric analysis; bold* ${ }^{*}$ significant correlation at $p \leq 0.05$. Table S4. Correlations among expression of TaCKX GFMs and NAC2 in seedling roots with seedling root weight and other yield-related traits with. ${ }^{1}$ - non-parametric analysis; bold* ${ }^{*}$ significant correlation at $p \leq 0.05$. Table S5. Correlations among expression of TaCKX GFMs and NAC2 in 7 DAP spikes from the growth chamber (A) and the field (B) and expression in seedling root. ' non-parametric analysis; bold* ${ }^{*}$ significant correlation at $p \leq 0.05$.

\section{Abbreviations}

CKX1-11: TaCKX1 - TaCKX11; NAC2: TaNAC2-5A; GFMs: Gene family members; GC: Growth chamber; CKX: Cytokinin oxidase/dehydrogenase; S: Plant Breeding Strzelce; D: Danko Hodowla Roslin; tZ: Trans-zeatin; iP: $N^{6}-(\Delta 2-$ isopentenyl) adenine; BA: Benzyladenine; TGW: Thousand grain weight

\section{Acknowledgements}

We thank Malgorzata Wojciechowska, Izabela Skuza and Agnieszka Glowacka for excellent technical assistance.

\section{Authors' contributions}

Conceptualization, A.N-O. and W.O.; methodology, K.S., H.O. and W.O. software, M.D-B.; validation, W.O.; formal analysis, K.S., M.D-B. and A.N-O.; investigation, K.S., H.O. and R.W.; resources, B.L. and B.Z.; data curation, K.S., H.O., B.L., B.Z. and R.W.; writing-original draft preparation, A.N-O.; writing-review and editing, A.N-O.; visualization, K.S. and A.N-O.; supervision, A.N-O.; project administration, A.N-O.; funding acquisition, A.N-O. All authors have read and agreed to the published version of the manuscript.

\section{Funding}

This research was supported by the Ministry of Agriculture and Rural Development, grant No. 5 PBwPR 4-1-01-4-02. The funding body had no role in the design of the study and collection, analysis, and interpretation of data and in writing the manuscript.

\section{Availability of data and materials}

All data generated or analysed during this study are included in this published article [and its supplementary information files].

Ethics approval and consent to participate

Not applicable.

\section{Consent for publication}

Not applicable.

\section{Competing interests}

The authors declare that they have no competing interests.

\section{Author details}

${ }^{1}$ Department of Functional Genomics, Plant Breeding and Acclimatization Institute - National Research Institute, Radzikow, 05-870 Blonie, Poland. ${ }^{2}$ Danko Hodowla Roslin Ltd., Choryn 27, 64-005 Koscian, Poland. ${ }^{3}$ Plant Breeding Strzelce Ltd., Co. - IHAR Group, Konczewice 1, 87-140 Chelmza, Poland. ${ }^{4}$ International Institute of Molecular and Cell Biology, Trojdena 4, 02-109 Warsaw, Poland. ${ }^{5}$ Department of Genetic Engineering, Plant Breeding and Acclimatization Institute - National Research Institute, Radzikow, 05-870 Blonie, Poland.

Received: 16 July 2020 Accepted: 20 October 2020

Published online: 29 October 2020

\section{References}

1. Schnurbusch T. Wheat and barley biology: towards new frontiers. J Integr Plant Biol. 2019;61(3):198-203.

2. Reynolds M, Braun H. WHEAT BREEDING benefits to low-input agriculture. Nat Plants. 2019;5(7):652-3.

3. Nadolska-Orczyk A, Rajchel IK, Orczyk W, Gasparis S. Major genes determining yield-related traits in wheat and barley. Theor Appl Genet. 2017;130(6):1081-98

4. Driever SM, Simkin AJ, Alotaibi S, Fisk SJ, Madgwick PJ, Sparks CA, Jones HD, Lawson T, Parry MAJ, Raines CA. Increased SBPase activity improves photosynthesis and grain yield in wheat grown in greenhouse conditions. Phil Trans R Soc B. 2017;372(1730):1-10.

5. Araus JL, Serret MD, Lopes MS. Transgenic solutions to increase yield and stability in wheat: shining hope or flash in the pan? J Exp Bot. 2019;70(5):1419-24.

6. Manners R, van Etten J. Are agricultural researchers working on the right crops to enable food and nutrition security under future climates? Global Environ Chang. 2018;53:182-94.

7. Werner T, Kollmer I, Bartrina I, Holst K, Schmulling T. New insights into the biology of cytokinin degradation. Plant Biol. 2006;8(3):371-81.

8. Jameson PE, Song JC. Cytokinin: a key driver of seed yield. J Exp Bot. 2016; 67(3):593-606

9. Kieber JJ, Schaller GE. Cytokinin signaling in plant development. Development. 2018;145(4):7.

10. Ashikari M, Sakakibara H, Lin SY, Yamamoto T, Takashi T, Nishimura A, Angeles ER, Qian Q, Kitano H, Matsuoka M. Cytokinin oxidase regulates rice grain production. Science. 2005;309(5735):741-5. 
11. Zalewski W, Galuszka P, Gasparis S, Orczyk W, Nadolska-Orczyk A. Silencing of the HvCKX1 gene decreases the cytokinin oxidase/dehydrogenase level in barley and leads to higher plant productivity. J Exp Bot. 2010;61(6):1839-51.

12. Zalewski W, Orczyk W, Gasparis S, Nadolska-Orczyk A. HvCKX2 gene silencing by biolistic or agrobacterium-mediated transformation in barley leads to different phenotypes. BMC Plant Biol. 2012;12:206.

13. Jablonski B, Ogonowska H, Szala K, Bajguz A, Orczyk W, Nadolska-Orczyk A. Silencing of TaCKX1 Mediates Expression of Other TaCKX Genes to Increase Yield Parameters in Wheat. Int J Mol Sci. 2020;21(13):1-21.

14. Argyros RD, Mathews DE, Chiang YH, Palmer CM, Thibault DM, Etheridge N, Argyros DA, Mason MG, Kieber JJ, Schaller GE. Type B response regulators of Arabidopsis play key roles in cytokinin signaling and plant development. Plant Cell. 2008;20(8):2102-16.

15. Mameaux S, Cockram J, Thiel T, Steuernagel B, Stein N, Taudien S, Jack P, Werner P, Gray JC, Greenland AJ, et al. Molecular, phylogenetic and comparative genomic analysis of the cytokinin oxidase/dehydrogenase gene family in the Poaceae. Plant Biotechnol J. 2012;10(1):67-82.

16. Ogonowska H, Barchacka K, Gasparis S, Jablonski B, Orczyk W, DmochowskaBoguta M, Nadolska-Orczyk A. Specificity of expression of TaCKX family genes in developing plants of wheat and their co-operation within and among organs. PLoS One. 2019;14(4):1-20.

17. Shoaib M, Yang WL, Shan QQ, Sajjad M, Zhang AM. Genome-wide identification and expression analysis of new cytokinin metabolic genes in bread wheat (Triticum aestivum L.). Peerj. 2019;7:1-23.

18. Song J, Jiang $L$, Jameson PE. Co-ordinate regulation of cytokinin gene family members during flag leaf and reproductive development in wheat. BMC Plant Biol. 2012;12:78.

19. Kersey PJ, Allen JE, Allot A, Barba M, Boddu S, Bolt BJ, Carvalho-Silva D, Christensen M, Davis P, Grabmueller C, et al. Ensembl genomes 2018: an integrated omics infrastructure for non-vertebrate species. Nucleic Acids Res. 2018:46(D1):D802-8.

20. Chen L, Zhao JQ, Song JC, Jameson PE. Cytokinin dehydrogenase: a genetic target for yield improvement in wheat. Plant Biotechnol J. 2020;18(3):614-30.

21. Zalewski W, Gasparis S, Boczkowska M, Rajchel IK, Kala M, Orczyk W, NadolskaOrczyk A. Expression patterns of HvCKX genes indicate their role in growth and reproductive development of barley. PLoS One. 2014;9(12):e115729.

22. Shanks CM, Hecker A, Cheng CY, Brand L, Collani S, Schmid M, Schaller GE, Wanke D, Harter K, Kieber JJ. Role of BASIC PENTACYSTEINE transcription factors in a subset of cytokinin signaling responses. Plant J. 2018;95(3):458-73.

23. He X, Qu BY, Li WJ, Zhao XQ, Teng W, Ma WY, Ren YZ, Li B, Li ZS, Tong YP. The nitrate-inducible NAC transcription factor TaNAC2-5A controls nitrate response and increases wheat yield. Plant Physiol. 2015;169(3):1991-2005.

24. Liang CZ, Wang YQ, Zhu YN, Tang JY, Hu B, Liu LC, Ou SJ, Wu HK, Sun XH, Chu JF, et al. OSNAP connects abscisic acid and leaf senescence by finetuning abscisic acid biosynthesis and directly targeting senescenceassociated genes in rice. P Natl Acad Sci USA. 2014;111(27):10013-8.

25. Redillas MCFR, Jeong JS, Kim YS, Jung H, Bang SW, Choi YD, Ha SH, Reuzeau C, Kim JK. The overexpression of OsNAC9 alters the root architecture of rice plants enhancing drought resistance and grain yield under field conditions. Plant Biotechnol J. 2012;10(7):792-805.

26. Qu BY, He X, Wang J, Zhao YY, Teng W, Shao A, Zhao XQ, Ma WY, Wang JY, Li $B$, et al. A wheat CCAAT box-binding transcription factor increases the grain yield of wheat with less fertilizer input. Plant Physiol. 2015;167(2):411-23.

27. Annunziata MG, Apelt F, Carillo P, Krause U, Feil R, Mengin V, Lauxmann MA, Kohl K, Nikoloski Z, Stitt M, et al. Getting back to nature: a reality check for experiments in controlled environments. J Exp Bot. 2017;68(16):4463-77.

28. Afsharyan NP, Sannemann W, Leon J, Ballvora A. Effect of epistasis and environment on flowering time in barley reveals a novel flowering-delaying OTL allele. J Exp Bot. 2020;71(3):893-906

29. Abid M, Shao YH, Liu SX, Wang F, Gao JW, Jiang D, Tian ZW, Dai TB. Predrought priming sustains grain development under post-anthesis drought stress by regulating the growth hormones in winter wheat (Triticum aestivum L.). Planta. 2017;246(3):509-24.

30. Liu Y, Gu DD, Wu W, Wen XX, Liao YC. The Relationship between Polyamines and Hormones in the Regulation of Wheat Grain Filling. PLoS One. 2013;8(10):1-10.

31. Yang LM, Fountain JC, Ji PS, Ni XZ, Chen SX, Lee RD, Kemerait RC, Guo BZ. Deciphering drought-induced metabolic responses and regulation in developing maize kernels. Plant Biotechnol J. 2018;16(9):1616-28.

32. Farooq $M$, Hussain $M$, Siddique KHM. Drought stress in wheat during flowering and grain-filling periods. Crit Rev Plant Sci. 2014;33(4):331-49.
33. Nelissen H, Sprenger H, Demuynck K, De Block J, Van Hautegem T, De Vliegher A, Inze D. From laboratory to field: yield stability and shade avoidance genes are massively differentially expressed in the field. Plant Biotechnol J. 2020;18(5):1112-4.

34. Ray R, Li DP, Halitschke R, Baldwin IT. Using natural variation to achieve a whole-plant functional understanding of the responses mediated by jasmonate signaling. Plant J. 2019;99(3):414-25.

35. Zhang L, Zhao YL, Gao LF, Zhao GY, Zhou RH, Zhang BS, Jia JZ. TaCKX6-D1, the ortholog of rice OsCKX2, is associated with grain weight in hexaploid wheat. New Phytol. 2012;195(3):574-84.

36. Lu J, Chang C, Zhang HP, Wang SX, Sun G, Xiao SH, Ma CX. Identification of a novel allele of TaCKX6a02 associated with grain size, filling rate and weight of common wheat. PLoS One. 2015;10(12):e0144765.

37. Chang C, Lu J, Zhang HP, Ma CX, Sun GL. Copy number variation of Cytokinin oxidase gene Tack $\times 4$ associated with grain weight and chlorophyll content of flag leaf in common wheat. PLoS One. 2015;10(12):15.

38. Schmulling T, Werner T, Riefler M, Krupkova E, Bartrina y Manns I. Structure and function of cytokinin oxidase/dehydrogenase genes of maize, rice, Arabidopsis and other species. J Plant Res. 2003;116(3):241-52.

39. Sorin C, Salla-Martret M, Bou-Torrent J, Roig-Villanova I, Martinez-Garcia JF. ATHB4, a regulator of shade avoidance, modulates hormone response in Arabidopsis seedlings. Plant J. 2009:59(2):266-77.

40. Argueso CT, Ferreira FJ, Kieber JJ. Environmental perception avenues: the interaction of cytokinin and environmental response pathways. Plant Cell Environ. 2009;32(9):1147-60.

41. Li YL, Song GQ, Gao J, Zhang SJ, Zhang RZ, Li W, Chen ML, Liu M, Xia XC, Risacher T, et al. Enhancement of grain number per spike by RNA interference of cytokinin oxidase 2 gene in bread wheat. Hereditas. 2018;155:1-8.

42. Werner T, Motyka V, Laucou V, Smets R, Van Onckelen H, Schmulling T. Cytokinin-deficient transgenic Arabidopsis plants show multiple developmental alterations indicating opposite functions of cytokinins in the regulation of shoot and root meristem activity. Plant Cell. 2003;15(11):2532-50.

43. Werner T, Schmulling T. Cytokinin action in plant development. Curr Opin Plant Biol. 2009;12(5):527-38.

44. Mrizova K, Jiskrova E, Vyroubalova S, Novak O, Ohnoutkova L, Pospisilova H, Frebort I, Harwood WA, Galuszka P. Overexpression of cytokinin dehydrogenase genes in barley (Hordeum vulgare cv. Golden Promise) fundamentally affects morphology and fertility. PLoS One. 2013;8(11):e79029.

45. Ramireddy E, Hosseini SA, Eggert K, Gillandt S, Gnad H, von Wiren N, Schmulling $T$. Root engineering in barley: increasing Cytokinin degradation produces a larger root system, mineral enrichment in the shoot and improved drought tolerance. Plant Physiol. 2018;177(3):1078-95.

46. Gao SP, Xiao YH, Xu F, Gao XK, Cao SY, Zhang FX, Wang GD, Sanders D, Chu CC. Cytokinin-dependent regulatory module underlies the maintenance of zinc nutrition in rice. New Phytol. 2019;224(1):202-15.

47. Zou X, Shao JW, Wang Q, Chen PS, Zhu YC, Yin CX. Supraoptimal Cytokinin Content Inhibits Rice Seminal Root Growth by Reducing Root Meristem Size and Cell Length via Increased Ethylene Content. Int J Mol Sci. 2018;19(12):1-16.

48. Gao SP, Fang J, Xu F, Wang W, Sun XH, Chu JF, Cai BD, Feng YQ, Chu CC. CYTOKININ OXIDASE/DEHYDROGENASE4 integrates Cytokinin and Auxin signaling to control Rice crown root formation. Plant Physiol. 2014;165(3):1035-46.

49. Mao CJ, He JM, Liu LN, Deng QM, Yao XF, Liu CM, Qiao YL, Li P, Ming F. OsNAC2 integrates auxin and cytokinin pathways to modulate rice root development. Plant Biotechnol J. 2020;18:429-42.

50. Nuccio ML, Paul M, Bate NJ, Cohn J, Cutler SR. Where are the drought tolerant crops? An assessment of more than two decades of plant biotechnology effort in crop improvement. Plant Sci. 2018;273:110-9.

51. Paolacci AR, Tanzarella OA, Porceddu E, Ciaffi M. Identification and validation of reference genes for quantitative RT-PCR normalization in wheat. BMC Mol Biol. 2009:10:11:1-27.

52. Frebort I, Sebela M, Galuszka P, Werner T, Schmulling T, Pec P. Cytokinin oxidase/cytokinin dehydrogenase assay: optimized procedures and applications. Anal Biochem. 2002;306(1):1-7.

53. Bradford MM, Williams WL. New, rapid, sensitive method for protein determination. Fed Proc. 1976:35(3):274

\section{Publisher's Note}

Springer Nature remains neutral with regard to jurisdictional claims in published maps and institutional affiliations. 